\title{
Tunneling and Filtering of Degenerate Microwave Modes in a Polarization-Dependent Waveguide Containing Index Gradient Barriers
}

\author{
A.B. Shvartsburg, ${ }^{1,2}$ S. Jiménez, ${ }^{3,{ }^{*}}$ N.S. Erokhin, ${ }^{4}$ and L. Vázquez ${ }^{5,6}$ \\ ${ }^{1}$ Joint Institute for High Temperatures Russian Academy of Sciences, Moscow \\ ${ }^{2}$ Institute for Space Researches Russian Academy of Sciences, Moscow \\ ${ }^{3}$ Universidad Politécnica de Madrid, Spain \\ ${ }^{4}$ Institute for Space Researches Russian Academy of Sciences, Moscow \\ ${ }^{5}$ Universidad Complutense de Madrid, Spain \\ ${ }^{6}$ Instituto de Matemática Interdisciplinar, Universidad Complutense de Madrid, Spain
}

(Received 21 December 2018; revised manuscript received 14 March 2019; published 17 April 2019)

\begin{abstract}
The drastic difference between complex transmission coefficients for $\mathrm{TE}_{11}$ and $\mathrm{TH}_{11} \mathrm{GHz}$ modes propagating through subwavelength gradient barriers in a metallic waveguide is examined. Amplitude-phase structures of the transmitted $\mathrm{TE}_{11}$ and $\mathrm{TH}_{11}$ modes are described in the framework of an exactly solvable model of these barriers. A sharp contrast in transmission is found between degenerate $\mathrm{TE}_{11}$ and $\mathrm{TH}_{11}$ modes distinguished by their polarization structures due to polarization-dependent tunneling and nonlocal energy transport through thin gradient dielectric barriers.
\end{abstract}

DOI: 10.1103/PhysRevApplied.11.044056

\section{INTRODUCTION}

This paper is aimed at controlling the reflectance and transmittance of microwaves in a waveguide loaded by specially designed inclusions. Some of these effects could be viewed as the microwave analogies of phenomena well known in optics and quantum mechanics, such as tunneling of microwave modes through opaque barriers formed by undersized segments of metallic waveguides [1,2]. These results provided the basis for the concept of reflectionless tunneling of waveguide modes in a diaphragm-loaded waveguide [3] expressed by plasmon-assisted transfer of wave energy through a diaphragm fabricated from a metamaterial [4]. This analogy was manifested by simulation of optical left-handed media realized in split-ring-resonatorloaded waveguides [5]. The growing applications of metamaterials in the design of waveguides gave rise to the concept of waveguide metatronics [6], opening new directions in waveguide functionality, such as the use of epsilon-near-zero metamaterials for squeezing energy flows [7-9], frequency-selective energy tunneling in wireloaded narrow waveguide channels [10,11], and waveguide structural dispersion [12]. Structural dispersion proved to be useful for controlling the dynamics of voltage and current waves in transmission lines with continuously distributed parameters [13]. Note that these phenomena were examined for the waveguide propagation of fundamental modes.

\footnotetext{
*s.jimenez@upm.es
}

In contrast, this paper focuses on the controlled transmission of higher transverse-electric $\mathrm{TE}_{11}$ and transverse-magnetic $\mathrm{TH}_{11}$ microwave modes through a gradient dielectric barrier in a waveguide. These modes have equal frequencies and the propagation constants form degenerate modes distinguished only by their polarization structures [14]. The appearance of degenerate modes poses the problem of filtering the flow of radiation, thereby weakening the undesirable "parasitic" mode. A simple example of such filtration, based on the different transmission of $\mathrm{TE}_{11}$ and $\mathrm{TH}_{11}$ modes through the heterogeneous dielectric nonmagnetic layer with a specially designed continuous distribution of dielectric permittivity located inside the waveguide, is examined below. This difference is determined by the polarization-dependent transmittance of these layers, fabricated from the dielectric without free carriers (gradient wave barriers).

Consider the transmittance spectra of microwave radiation with frequency $\omega$ propagating in a rectangular metallic waveguide with sides $a$ and $b(a>b)$ containing gradient dielectric layers (Fig. 1); these spectra depend upon the distribution of dielectric permittivity $\varepsilon(z)$ inside the layers disposed along the waveguide axis ( $z$ direction). The $x$ and $y$ axes are parallel to the waveguide cross section with sides $a$ and $b$ respectively. The sides of the gradient layer, located in the $x-y$ plane, are also equal to $a$ and $b$; these layers can be viewed as gradient wave barriers. The transmittance spectra of both single-layer and multilayer gradient structures are examined below by means of exact analytical solutions of Maxwell's equations 


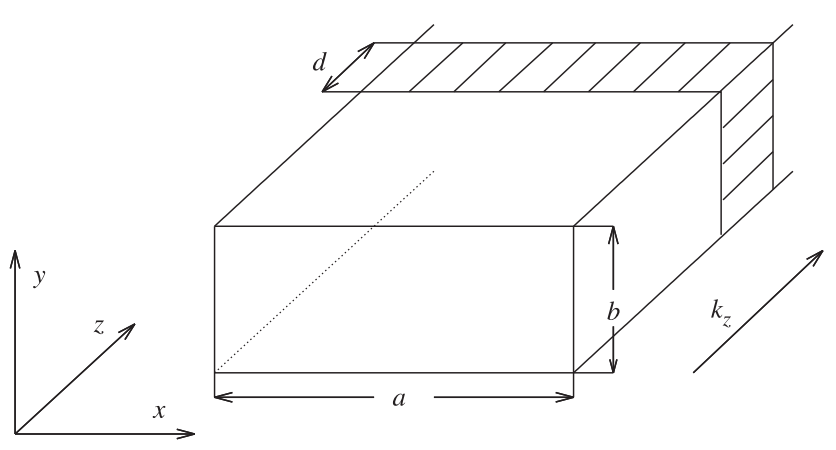

FIG. 1. Geometry of the problem. Microwaves are traveling in the $z$ direction along the rectangular waveguide with sides $a$ and $b$; the $x$ and $y$ axes are oriented along these sides. Gradient dielectric barriers with thickness $d$ are disposed across the waveguide.

for isotropic nonmagnetized lossless media; these solutions are obtained independently of any suppositions about smallness or slowness of variations of fields or the spatial distributions: $\varepsilon(z)$ :

$\operatorname{rot} \vec{E}=-\frac{1}{c} \frac{\partial \vec{H}}{\partial t} ; \quad \operatorname{rot} \vec{H}=\frac{1}{c} \frac{\partial \vec{D}}{\partial t} ; \operatorname{div} \vec{D}=0 ; \quad \operatorname{div} \vec{H}=0$

The electric displacement $\vec{D}$ and electric field $\vec{E}$ are linked by the usual correlation $\vec{D}=\varepsilon(z) \vec{E}$. To describe the fields of $\mathrm{TE}_{11}$ and $\mathrm{TH}_{11}$ modes in the rectangular waveguide, it is expedient to introduce the generating functions presented by vectors $\vec{A}=\left(A_{x}, A_{y}, 0\right)$ and $\vec{P}=\left(P_{x}, P_{y}, 0\right)$. The transverse-electric $\vec{E}$ and transverse-magnetic $\vec{H}$ components of these modes, located in the $x-y$ plane, are expressed via the vectors $\vec{A}$ and $\vec{P}$, respectively:

$$
\begin{gathered}
\mathrm{TE}_{11} \text { mode: } \vec{E}=-\frac{1}{c} \frac{\partial \vec{A}}{\partial t} ; \quad \vec{H}=\operatorname{rot} \vec{A} ; \\
\mathrm{TH}_{11} \text { mode: } \vec{H}=-\frac{1}{c} \frac{\partial \vec{P}}{\partial t} ; \quad \vec{E}=\frac{\operatorname{rot} \vec{P}}{\varepsilon(z)} .
\end{gathered}
$$

From Maxwell's equations (1), we have that vectors $\vec{A}$ and $\vec{P}$ are governed by

$$
\operatorname{rot} \operatorname{rot} \vec{A}+\frac{\varepsilon(z)}{c^{2}} \frac{\partial^{2} \vec{A}}{\partial t^{2}}=0, \quad \operatorname{rot} \frac{\operatorname{rot} \vec{P}}{\varepsilon(z)}+\frac{1}{c^{2}} \frac{\partial^{2} \vec{A}}{\partial t^{2}}=0 .
$$

The electric displacement $\vec{D}$ and electric field $\vec{E}$ are linked by $\vec{D}=\varepsilon(z) \vec{E}$. Omitting the phase factor $\exp (-i \omega t)$ for simplicity, supposing the waveguide walls to be perfectly conducting, and using the boundary conditions for the tangent components of the electric fields on these walls, $\left.E_{\text {tg }}\right|_{x=0, a}=\left.E_{\text {tg }}\right|_{y=0, b}=0$, one can present the components of the generating vector functions $\vec{A}$ and $\vec{P}$ as

$$
\begin{cases}A_{x}=A_{1} \varrho_{1}(x, y) \Psi_{s}(z), & A_{y}=A_{2} \varrho_{2}(x, y) \Psi_{s}(z), \\ P_{x}=P_{1} \varrho_{2}(x, y) \Psi_{p}(z), & P_{y}=P_{2} \varrho_{1}(x, y) \Psi_{p}(z) .\end{cases}
$$

The functions $\varrho_{1}$ and $\varrho_{2}$ in Eq. (4) provide the well-known expressions for the components of the $\mathrm{TE}_{11}$ and $\mathrm{TH}_{11}$ modes in a rectangular waveguide [14]:

$$
\begin{aligned}
& \varrho_{1}(x, y)=\cos \left(\frac{\pi x}{a}\right) \sin \left(\frac{\pi y}{b}\right), \\
& \varrho_{2}(x, y)=\sin \left(\frac{\pi x}{a}\right) \cos \left(\frac{\pi y}{b}\right),
\end{aligned}
$$

where coefficients $A_{1,2}$ and $P_{1,2}$ are the normalization constants. The coefficients $A_{1,2}$ as well as $P_{1,2}$ are linked: thus, in the case of the $\mathrm{TE}_{11}$ mode $\left(E_{z}=0\right)$, substitution of the generating function in Eq. (4) into Eq. (2) gives the values of components $H_{x}$ and $H_{y}$. Further substitution of these components into the equation $\operatorname{div} \vec{D}=0$ gives the correlation

$$
b A_{1}+a A_{2}=0 .
$$

Similar operations for the $\mathrm{TH}_{11}$ mode $\left(H_{z}=0\right)$ and the equation $\operatorname{div} \vec{H}=0$ provide the link

$$
b P_{1}+a P_{2}=0 .
$$

The functions $\Psi_{s}(z)$ and $\Psi_{p}(z)$ represent the variation of the $\mathrm{TE}_{11}$ and $\mathrm{TH}_{11}$ modes upon the $z$ coordinate. Outside the gradient structure, these functions are given by the harmonic waves [14]

$$
\begin{aligned}
\Psi_{s}(z) & =\Psi_{p}(z)=\exp \left( \pm i k_{z} z\right), \quad k_{z}=\sqrt{\frac{\omega^{2}}{c^{2}}-k_{\perp}^{2}}, \\
k_{\perp}^{2} & =\pi^{2}\left(\frac{1}{a^{2}}+\frac{1}{b^{2}}\right) .
\end{aligned}
$$

Both $\mathrm{TE}_{11}$ and $\mathrm{TH}_{11}$ modes have equal cutoff frequencies $\omega_{1}=k_{\perp} c$.

Inside the gradient structures, these functions $\Psi_{s}(z)$ and $\Psi_{p}(z)$ depend on the spatial distribution of the dielectric permittivity $\varepsilon(z)$. To highlight the effects associated with the nonuniformity of $\varepsilon$, we assume that wave absorption and material dispersion are insignificant in the range of microwave frequencies $\omega$ under consideration. In this case, the spatial dependence of the isotropic dielectric permittivity in the layer, $\varepsilon(z)$, can be written as

$$
\varepsilon(z)=n_{0}^{2} U^{2}(z),\left.\quad U\right|_{z=0}=1 .
$$

Here, $n_{0}$ is the refractive index of the layer at the boundary $z=0$ and the dimensionless function $U^{2}(z)$ describes the 
normalized spatial variation of the permittivity. Gradient dielectric layers with fixed profiles $U^{2}(z)$ can be fabricated from the porous dielectrics with a controlled distribution of air-filled pores [15-17]. Using Eq. (9) and substituting Eqs. (2)-(5) into Maxwell's equations (1) gives the pair of equations that govern the unknown functions $\Psi_{s}(z)$ and $\Psi_{p}(z)$ :

$$
\begin{gathered}
\frac{d^{2} \Psi_{s}}{d z^{2}}+\left[\frac{\omega^{2} n_{0}^{2} U^{2}(z)}{c^{2}}-k_{\perp}^{2}\right] \psi_{s}=0, \\
\frac{d^{2} \Psi_{p}}{d z^{2}}+\left[\frac{\omega^{2} n_{0}^{2} U^{2}(z)}{c^{2}}-k_{\perp}^{2}\right] \psi_{p}=\frac{2}{U(z)} \frac{d U(z)}{d z} \frac{d \Psi_{p}}{d z} .
\end{gathered}
$$

Note that the functions $\Psi_{s}(z)$ and $\Psi_{p}(z)$, related to the degenerate $\mathrm{TE}_{11}$ and $\mathrm{TH}_{11}$ modes in the same waveguide, are determined by two different equations (10) and (11). The flexible distribution of the refractive index (9), containing two free parameters, is shown below in Sec. II to reduce the set in Eqs. (10) and (11) to a pair of similar master equations. The amplitude-phase transmittance spectra based on the exact analytical solutions of the master equations for $\mathrm{TE}_{11}$ and $\mathrm{TH}_{11}$ modes traveling through the gradient barriers $U(z)$ are presented in Sec. III: here, we illustrate the possibility of effectively controlled quenching and filtration of these degenerate modes due to the drastic difference between their polarization-dependent transmittance. The main results of this analysis are summarized in the conclusion (Sec. IV). To obtain the solutions of the master equations and find the amplitude-phase transmittance spectra for degenerate $\mathrm{TE}_{11}$ and $\mathrm{TH}_{11}$ modes, some tedious algebra is needed: the corresponding calculations are removed to the appendixes.

\section{EXACTLY SOLVABLE MODEL OF GRADIENT BARRIER IN A WAVEGUIDE}

It is convenient to reduce Eqs. (10) and (11) to a single equation with different parameters. By introducing the new functions $f_{s}$ and $f_{p}$ and a new variable $\eta$,

$$
f_{s}=\Psi_{s} \sqrt{U}, \quad f_{p}=\frac{\Psi_{p}}{\sqrt{U}}, \quad \eta=\int_{0}^{z} U\left(z_{1}\right) d z_{1},
$$

one can represent Eqs. (10) and (11) for the $\mathrm{TE}_{11}$ and $\mathrm{TH}_{11}$ modes in a similar form:

$$
\begin{aligned}
& \frac{d^{2} f_{s}}{d \eta^{2}}+f_{s}\left(K-\frac{U_{\eta \eta}}{2 U}+\frac{U_{\eta}^{2}}{4 U^{2}}\right)=0, \\
& \frac{d^{2} f_{p}}{d \eta^{2}}+f_{p}\left(K+\frac{U_{\eta \eta}}{2 U}-\frac{3 U_{\eta}^{2}}{4 U^{2}}\right)=0,
\end{aligned}
$$

$$
K=\left(\frac{\omega n_{0}}{c}\right)^{2}-\frac{k_{\perp}^{2}}{U^{2}(z)}, \quad U_{\eta}=\frac{d U}{d \eta}, \quad U_{\eta \eta}=\frac{d^{2} U}{d \eta^{2}}
$$

Equations (10) and (11) and Eqs. (13) and (14) are valid for the arbitrary profiles $U(z)$ and $U(\eta)$, respectively. However, in our problem, one has to consider the profiles $U(z)$, ensuring the exact analytical solutions for both Eqs. (10) and (11). One of these profiles is given by the so-called, Rayleigh distribution $U(z)=(1+z / L)^{-1}$ [18], containing only one free parameter: the length scale $L$. In terms of the variable $\eta$ defined in Eq. (12), this distribution can be expressed as $U(\eta)=\exp (-\eta / L)$. Substituting this into Eqs. (13) and (14) results in the same equations for both the $\mathrm{TE}_{11}$ and $\mathrm{TH}_{11}$ modes and, therefore, this distribution is invalid for filtration of these degenerate modes. In contrast, the flexible distribution

$$
U(z)=\left[\cos \left(\frac{z}{L}\right)+M \sin \left(\frac{z}{L}\right)\right]^{-1},
$$

containing two free parameters, $L$ and $M$, is shown below to provide an essential difference in transmission for the degenerate modes suitable for their effective filtration. The values of $L$ and $M$ depend on the minimum of the refractive index, $n_{m}$, and on the layer thickness $d$. Considering the symmetrical profile $U(0)=U(d)=1$ (Fig. 2), one can link these quantities to the physical and geometrical parameters of the layer:

$$
n_{m}=\frac{n_{0}}{\sqrt{1+M^{2}}}, \quad \frac{d}{L}=2 \arctan M
$$

Substituting the distribution (16) into the definition of the new variable $\eta$ in Eq. (12) yields the link between

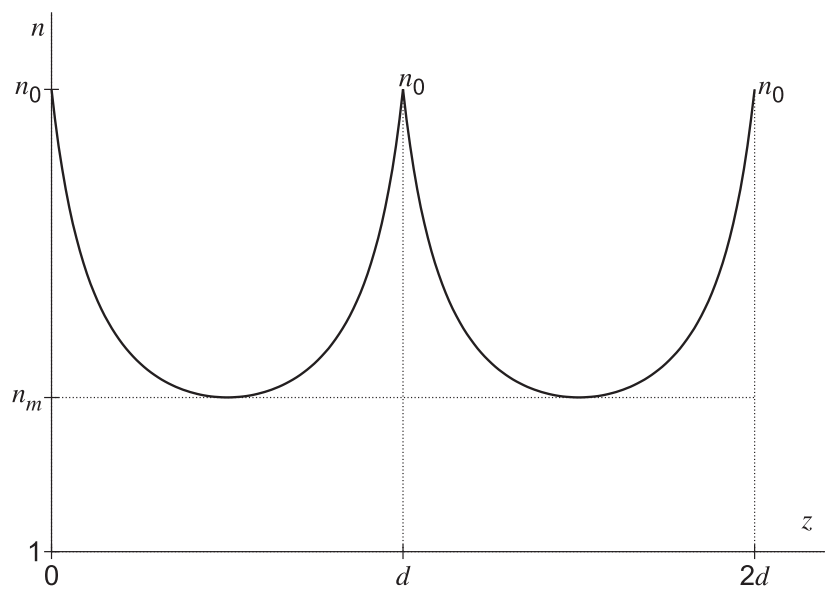

FIG. 2. Geometry of the problem: distribution of the refractive index $n(z)=n_{0} U(z)$ with $U$ given by Eq. (16) across the adjoining barriers. The values $n_{0}$ and $n_{m}$ correspond, respectively, to the boundaries of each barrier and to the minimum value of $n(z)$. 
variables $\eta$ and $z$ :

$$
\begin{aligned}
\frac{\eta}{L} & =\frac{1}{\sqrt{1+M^{2}}} \ln \left(\frac{1+m_{+} \tan \left(\frac{z}{2 L}\right)}{1-m_{-} \tan \left(\frac{z}{2 L}\right)}\right), \\
m_{ \pm} & =\sqrt{1+M^{2}} \pm M .
\end{aligned}
$$

Introducing a new variable $\varsigma$, determined in the interval $-\varsigma_{0} \leq \varsigma \leq \varsigma_{0}$, corresponding to the values $0 \leq z \leq d$,

$$
\begin{aligned}
\varsigma & =\frac{\eta \sqrt{1+M^{2}}}{L}-\varsigma_{0}, \quad \varsigma_{0}=\ln \left(m_{+}\right), \\
\varsigma_{\left.\right|_{z=0}} & =-\varsigma_{0}, \quad \varsigma_{\left.\right|_{z=d}}=\varsigma_{0},
\end{aligned}
$$

one can write the distribution function $U(z)$ (16) via the new variable $\varsigma$ :

$$
U(\varsigma)=\frac{\cosh (\varsigma)}{\sqrt{1+M^{2}}}, \quad U\left(\varsigma_{0}\right)=U\left(-\varsigma_{0}\right)=1 .
$$

Consider the propagating modes incident on the interface $(z=0)$ of the gradient layer (16). To obtain an exact analytical solution to the wave equations (13) and (14) for this barrier, one needs to rewrite these equations by introducing the new variable $\varsigma$ defined in Eq. (19). It is noteworthy that, once these transformations are made, the equations governing the functions $f_{s}$ and $f_{p}$ can be presented in the same form:

$$
\frac{d^{2} f_{s, p}}{d \varsigma^{2}}+f_{s, p}\left(h^{2}-\frac{\aleph_{s, p}}{\cosh ^{2}(\varsigma)}\right)=0 .
$$

Note that Eq. (21) is formally identical to the onedimensional, time-dependent, Schrödinger equation with the potential $\cosh ^{-2}(\varsigma)$ [19]. The values of the parameter $h^{2}$ in Eq. (21) are equal for Eqs. (13) and (14), governing the $f_{s}$ and $f_{p}$ functions, while the parameters $\aleph_{s}$ and $\aleph_{p}$ are related to functions $f_{s}$ and $f_{p}$, respectively:

$$
\begin{aligned}
h^{2} & =\frac{1}{1+M^{2}}\left(\frac{\omega n_{0} L}{c}\right)^{2}-\frac{1}{4}, \quad \aleph_{s}=\left(k_{\perp} L\right)^{2}+\frac{1}{4}, \\
\aleph_{p} & =\left(k_{\perp} L\right)^{2}-\frac{3}{4} .
\end{aligned}
$$

Because of the expressions (21) and (22), the forthcoming analysis of polarization-dependent transmittance of different modes can be unified.

\section{CONTROLLED QUENCHING AND FILTRATION OF DEGENERATE MODES PASSING THROUGH GRADIENT DIELECTRIC STRUCTURES IN A WAVEGUIDE}

To visualize the amplitude-phase spectra of the transmitted $\mathrm{TE}_{11}$ (B11) and $\mathrm{TH}_{11}$ (C7) modes, it is convenient to introduce the characteristic frequency $\Omega$ and the dimensionless parameter $u$ :

$$
\Omega=\frac{c \sqrt{1+M^{2}}}{2 n_{0} L}, \quad u=\frac{\Omega}{\omega} .
$$

Now it is worthwhile to rewrite Eq. (21) by expressing the term $h^{2}$ (22) through the frequency-dependent parameter $u$ :

$$
\frac{d^{2} f_{s, p}}{d \varsigma^{2}}+f_{s, p}\left[\frac{1}{4}\left(\frac{1}{u^{2}}-1\right)-\frac{\aleph_{s, p}}{\cosh ^{2}(\varsigma)}\right]=0 .
$$

The condition of absolute convergence of solutions of Eq. (21), $h^{2} \leq 0$ (see Appendix A), implies the restriction of values $u$ in Eq. (24): $u \leq 1(\omega<\Omega)$. Thus, the expression in the square brackets in Eq. (24) for the function $f_{s}$ (the $\mathrm{TE}_{11}$ mode) is always negative. Meanwhile, for the function $f_{p}$ (the $\mathrm{TH}_{11}$ mode), this expression can become positive. This result means that the $\mathrm{TE}_{11}$ mode is always passing through the gradient layer (16) in the tunneling regime, while the $\mathrm{TH}_{11}$ mode can travel through the same layer in the propagating regime. This case, which provides a strong contrast between the transmitted $\mathrm{TE}_{11}$ and $\mathrm{TH}_{11}$ modes, is examined below.

Consider, for instance, a waveguide with sides $a=2 \mathrm{~cm}$ and $b=1 \mathrm{~cm}$. The cutoff frequency for these modes is [14] $\omega_{1}=0.5 \pi c^{-1} \sqrt{a^{2}+b^{2}}=10.53 \times 10^{10} \mathrm{rad} / \mathrm{s}(16.77$ $\mathrm{GHz}$ ). On the other hand, considering the parameters of the gradient layer to be $n_{0}=2, M=1$, and $L=0.075 \mathrm{~cm}$, we obtain the characteristic frequency $\Omega=14.142 \times 10^{10}$ $\mathrm{rad} / \mathrm{s}(22.52 \mathrm{GHz})$. Recalling the condition for $\mathrm{TE}_{11}$ and $\mathrm{TH}_{11}$ modes to propagate $\omega>\omega_{1}=10.53 \times 10^{10} \mathrm{rad} / \mathrm{s}$, one can determine the spectral range of the modes discussed: $1<u<1.343$; all the spectra considered here are computed inside this range. Some salient features of the interaction of $\mathrm{TE}_{11}$ and $\mathrm{TH}_{11}$ modes with the gradient dielectric barriers are listed below.

(1) The amplitude-phase transmittance spectra for degenerated $\mathrm{TE}_{11}$ and $\mathrm{TH}_{11}$ modes passing through a single gradient layer and computed by means of the formulas (B11) and (C7), respectively, are presented in Figs. 3 and 4.

Figure 3 shows that the transmittance of the $\mathrm{TE}_{11}$ mode exceeds the transmittance of the $\mathrm{TH}_{11}$ mode over the entire spectral range $1<u<1.343$. Thus, in the case $u=1.15$, the value of the transmission coefficient $|T|^{2}$ for mode $\mathrm{TE}_{11}$ is twice the value of $|T|^{2}$ for mode $\mathrm{TH}_{11}$.

The transmittance of both modes vanishes $\left(|T|^{2} \rightarrow 0\right)$ near the cutoff frequency $u \rightarrow 1.343$. The phase of the traveling $\mathrm{TH}_{11}$ mode (Fig. 4, curve 2 ) is characterized by an effective frequency dispersion, while the dependence of the phase of the tunneling mode $\mathrm{TE}_{11}$ on the frequency is weak (Fig. 4, curve 1).

(2) The difference in the transmittance of the degenerate modes passing through the single gradient layer is 


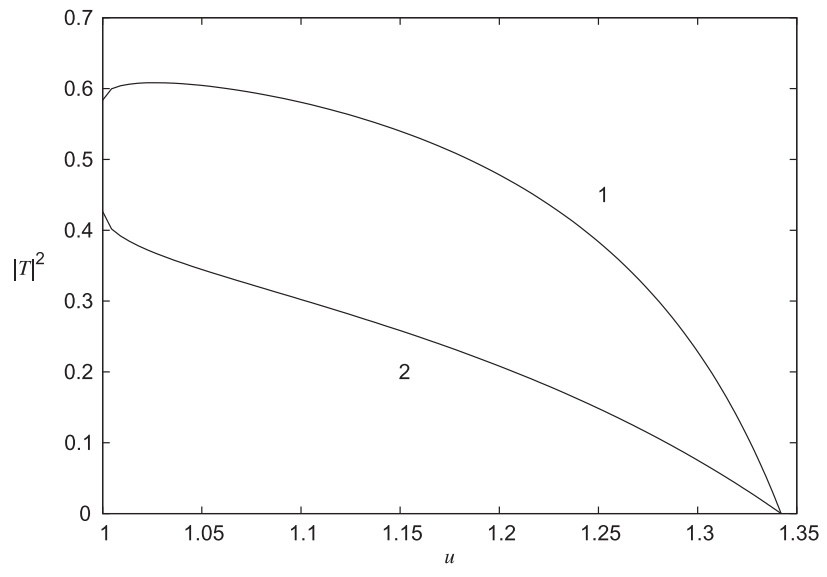

FIG. 3. Transmittance spectra of the degenerate $\mathrm{TE}_{11}$ and $\mathrm{TH}_{11}$ modes propagating in a waveguide with sides $a=2 \mathrm{~cm}, b=1$ $\mathrm{cm}$ (as represented in Fig. 1) through a single subwavelength gradient barrier (16) with thickness $d=0.115 \mathrm{~cm}$ and parameters $n_{0}=2, M=1$. The transmission coefficients with respect to the energy, $|T|^{2}$, of the transmitted waves are plotted vs the normalized frequency $u$ (23). Curves 1 and 2 correspond to the $\mathrm{TE}_{11}$ and $\mathrm{TH}_{11}$ modes, respectively.

increased drastically due to the use of two similar adjoining barriers (Figs. 5 and 6).

For the frequency $u=1.15$, one can see in Fig. 3 that the value of the transmission coefficient $|T|^{2}$ for the $\mathrm{TE}_{11}$ mode (Fig. 5) exceeds the value of $|T|^{2}$ for the $\mathrm{TH}_{11}$ mode by a factor of 32 . Unlike the monotonic variation of the phase for the traveling $\mathrm{TH}_{11}$ mode (Fig. 6), the phase of the tunneling $\mathrm{TE}_{11}$ mode tends to saturation. This feature is characteristic of tunneling [2].

To visualize the effect of the gradient profile (16), the transmission coefficients for the $\mathrm{TE}_{11}$ and $\mathrm{TH}_{11}$ modes passing through the homogeneous dielectric layer with

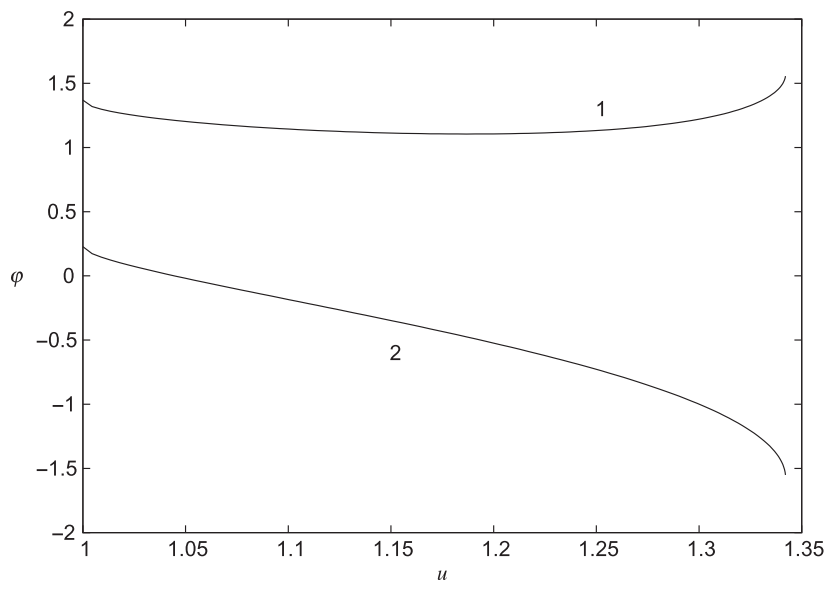

FIG. 4. Same conditions as in Fig. 3. The phases of the transmission coefficients, $\varphi$, for the transmitted waves are plotted vs the normalized frequency $u$ (23). Curves 1 and 2 correspond to the $\mathrm{TE}_{11}$ and $\mathrm{TH}_{11}$ modes, respectively.

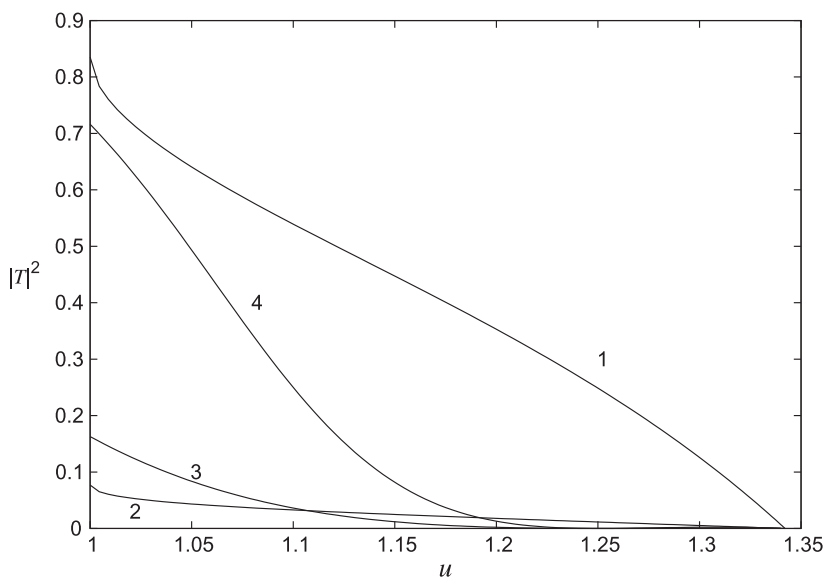

FIG. 5. Transmittance spectra of the degenerate $\mathrm{TE}_{11}$ and $\mathrm{TH}_{11}$ modes propagating through a pair of adjoining gradient barriers (16) with the same parameters as in Fig. 3. The transmission coefficients, $|T|^{2}$, of the transmitted waves are plotted vs the normalized frequency $u(23)$. Curves 1 and 2 correspond to the $\mathrm{TE}_{11}$ and $\mathrm{TH}_{11}$ modes, respectively. Curves 3 and 4 represent the transmission coefficients $|T|^{2}$ for the propagation of the same modes through the homogeneous layer with the same refractive index $n_{0}=2$ and thickness $d=0.23 \mathrm{~cm}$.

the same refractive index $n_{0}$ and the same thickness $2 d$ are presented in Fig. 5 by spectra 3 and 4 respectively. Comparison of these curves with spectra 1 and 2 shows that, unlike in the gradient barriers, transmittance of the $\mathrm{TH}_{11}$ mode through the homogeneous barriers exceeds the transmittance of the $\mathrm{TE}_{11}$ mode. However, the physically meaningful difference in the transmittance of these modes is manifested in the spectral range $1<u<1.2$ only; the values of $|T|^{2}$ for both modes become less than $1 \%$ for the smaller frequencies $u \geq 1.2$.

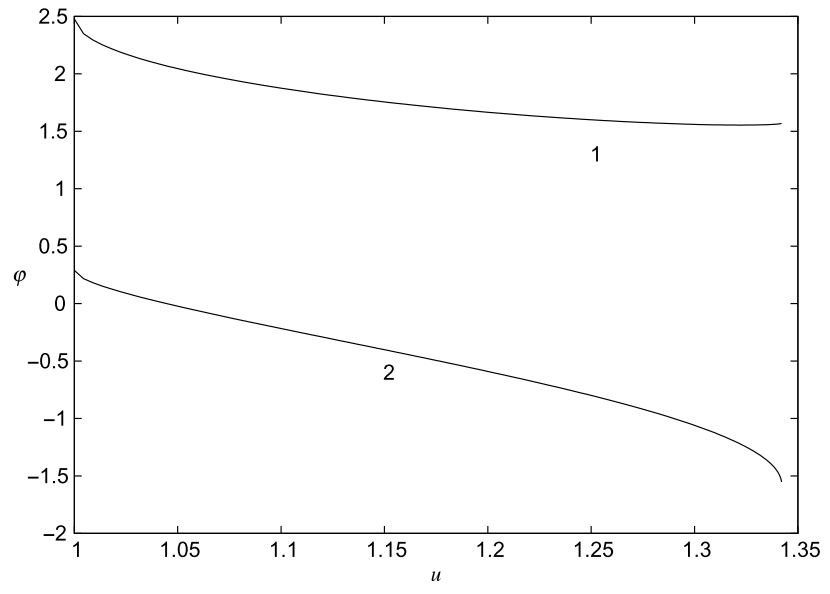

FIG. 6. Same conditions as in Fig. 5. The phases of the transmission coefficients, $\varphi$, for the transmitted waves are plotted vs the normalized frequency $u$ (23). Curves 1 and 2 correspond to the $\mathrm{TE}_{11}$ and $\mathrm{TH}_{11}$ modes, respectively. 
(3) The attenuation of the transmitted $\mathrm{TE}_{11}$ mode due to its reflectance and tunneling is much less than the attenuation of the transmitted $\mathrm{TH}_{11}$ mode caused by reflectance alone. This effect can be understood as the first stage of reflectionless tunneling of electromagnetic waves through the set of gradient barriers [16], when the high transmittance is determined mainly by the nonlocal energy transport by the tunneling waves $\left(|T|^{2} \approx 0.9--0.95\right)$.

(4) Note that the thickness $d=1.15 \mathrm{~mm}$ (17) of the barrier in this entire spectral range $\omega_{1}<\omega<\Omega$ is much smaller than the wavelengths of the traveling modes: $\lambda \geq 2$ $\mathrm{cm}$. The absorption of microwaves in these thin subwavelength nonconducting layers is negligible. The quenching of the transmitted $\mathrm{TH}_{11}$ mode is provided not by the absorption in the barrier, but by its reflection from the barrier.

(5) The same analysis can be carried out for any other pair of degenerate $\mathrm{TE}_{m j}$ and $\mathrm{TH}_{m j}$ modes. To perform this generalization, one has to make the replacements $x \rightarrow m x$ and $y \rightarrow j y$ in functions $\varrho_{1}$ and $\varrho_{2}$ (4) that describe the spatial structure of these modes and to consider the solutions in the spectral range between the characteristic frequency $\Omega$ (23) and the cutoff frequency of the $\mathrm{TE}_{m j}$ and $\mathrm{TH}_{m j}$ modes.

Thus, even a pair of subwavelength gradient barriers ensures strong filtering of the degenerate modes, providing high contrast between the power flows in the transmitted $\mathrm{TE}_{11}$ and $\mathrm{TH}_{11}$ modes.

The analysis of the filtering of degenerate modes is presented here for the cases of one and two gradient layers. However, even these simple barriers are shown to provide a difference in the transmittance of the degenerate $\mathrm{TE}_{11}$ and $\mathrm{TH}_{11}$ modes by more than a factor of 30 . If necessary for any concrete problem, the complex generalized formulas for the reflection and/or transmission coefficients for three or more layers, based on massive algebra, can be derived by means of the computational procedure presented for two layers in Appendix D.

\section{CONCLUSION}

In conclusion, we consider the possibility of controlled formation of amplitude-phase spectra of transverse-electric (TE) and transverse-magnetic (TH) modes transmitted through gradient dielectric lossless layers in a waveguide. The nonlocal dispersion of these modes inside the gradient layers, determined by the spatial distribution of the dielectric permittivity inside each layer and its thickness, determines the contrast in the transmittance of the modes discussed. This contrast is shown to ensure the effective quenching and filtering of the degenerate $\mathrm{TE}_{11}$ and $\mathrm{TH}_{11}$, distinguished only by their polarization structures. Some salient features of this polarization-dependent effect are listed below. (i) Owing to the smooth, continuously shaped distribution of dielectric permittivity across the gradient layer, these thin subwavelength layers are shown to provide flexible control over the spectra of the transmitted modes.

(ii) The propagation of the $\mathrm{TE}_{11}$ mode through the gradient layer is an example of tunneling of waves with vector polarization structure characterized by one longitudinal $\left(H_{z}\right)$ and four transverse $\left(E_{x}, E_{y}, H_{x}, H_{y}\right)$ components. Unlike the well-known effect of frustrated total internal reflection of both TE- and TH-polarized waves in a homogeneous opaque medium, this polarization-dependent tunneling determined by the nonlocal dispersion of the gradient layer arises only for the TE-polarized mode in the exactly solvable model of a gradient layer (16).

(iii) Exact analytical solutions of Eqs. (10) and (11) for the model (16) presented by the expressions (B1) and (C1), respectively, are obtained without any suppositions about smallness or slowness of the spatial variations of the dielectric permittivity $U(z)$ inside the layer. These solutions may become useful for several problems of crossdisciplinary physics dealing with the incidence of waves of different physical nature on the interface of layered media at an arbitrary angle. Unlike widely used models of these media built from broken lines (boxlike [20] or "trapezoidal" [21] potentials), the smooth flexible distribution of potential (16) may pave the way to revisit the series of reflectance and/or transmittance problems in optics [22], acoustics [23], electromagnetics of heterogeneous media [24], and matter waves physics [25].

\section{ACKNOWLEDGMENTS}

The authors thank Professor O.F. Petrov for the support of this research. S.J. and L.V. thank the partial support of the Spanish Ministerio de Economía y Empresa under Grant No. ESP2016-79135-R. A.S. conceived the idea and supervised the project, N.E. conducted the initial study of microwaves in the heterogeneous media, and S.J. and L.V. carried out the computations. All authors contributed to interpreting the results and writing the manuscript.

\section{APPENDIX A: SOLUTION OF THE MASTER EQUATIONS}

To find the solutions of Eq. (21), it is worthwhile to introduce a new variable $v$ and a new function $F(v)$, given by

$$
v=\frac{1-\tanh (\varsigma)}{2}, \quad f(\varsigma)=\frac{1}{\cosh ^{2}(\varsigma)} F(v)
$$


After this transformation, the equation governing the function becomes

$$
\nu(1-\nu) \frac{d F^{2}}{d \nu^{2}}+[\gamma-(1+\alpha+\beta) \nu] \frac{d F}{d \nu}-\alpha \beta F=0 .
$$

Equation (A2) is the hypergeometric equation in its standard form, where the parameters $\alpha, \beta$, and $\gamma$ are defined via the quantities $h^{2}$ and $\aleph_{s, p}(22)$ :

$$
\begin{gathered}
\gamma=1+2 p, \quad 2 p= \pm \sqrt{-h^{2}}, \\
\alpha_{s}, \beta_{s}=2 p+\frac{1}{2} \pm i k_{\perp} L, \\
\alpha_{p}, \beta_{p}=2 p+\frac{1}{2} \pm \sqrt{1-\left(k_{\perp} L\right)^{2}} .
\end{gathered}
$$

Thus, the values of the parameters $\alpha$ and $\beta$ are different for the $\mathrm{TE}_{11}$ and $\mathrm{TH}_{11}$ modes, while the parameter $\gamma$ has the same value for both modes. The hypergeometric equation (A2) has two linearly independent solutions $F_{1}$ and $F_{2}$. Since the parameters $\alpha, \beta$, and $\gamma$ are linked by the relation $\operatorname{Re}(\alpha+\beta+1)=2 \gamma$, these solutions are given by the hypergeometric series $G$ [26]:

$$
F_{1}=G(\alpha, \beta, \gamma, \nu), \quad F_{2}=G(\alpha, \beta, \gamma, 1-\nu) .
$$

The hypergeometric series $G$ are known to converge absolutely inside the circle $|v|=1$ under the condition $\operatorname{Re}(\alpha+\beta-\gamma)<0$ [26]. According to Eqs. (A3) and (A4), this process corresponds to $\alpha+\beta-\gamma=2 p$. This result means that the series (A5) converge absolutely if the value $p$ is negative, which means, in turn, that the condition $h^{2}<0$ has to be satisfied. This case is considered below.

Using a standard procedure to find the reflection and/or transmission coefficients for the barrier (16) based on the continuity conditions of the components of the modes on the boundaries $z=0$ and $z=d$ of the gradient layer, the values of the variables $\varsigma$ and $v$ at these boundaries can be shown to satisfy

$$
\begin{gathered}
z=0: \quad \varsigma=-\ln \left(m_{+}\right), \quad v_{ \pm}=\frac{1}{2}\left(1 \pm \frac{M}{\sqrt{1+M^{2}}}\right), \\
\cosh (\varsigma)=\sqrt{1+M^{2}}, \\
\tanh (\varsigma)=-\frac{M}{\sqrt{1+M^{2}}}, \quad \text { (A6) } \\
z=d: \quad \varsigma=\ln \left(m_{+}\right), \quad v=v_{-}, \quad h=v_{+}, \\
\cosh (\varsigma)=\sqrt{1+M^{2}}, \quad \tanh (\varsigma)=\frac{M}{\sqrt{1+M^{2}}} . \quad \text { (A7) }
\end{gathered}
$$

Since the transformations (12) ensure the presentation of the equations for both the $S$ - and the $P$-polarized waves under the same form (21), the following analysis of polarization effects proves to be standardized. The continuity conditions on the boundaries of the gradient layers provide the essential difference in the reflectance and/or transmittance spectra for the $\mathrm{TE}_{11}$ and $\mathrm{TH}_{11}$ modes. Although we achieve a single formulation for both cases, it is convenient, in order to simplify the computations, to examine the spectra separately.

\section{APPENDIX B: PROPAGATION OF THE TE 11 MODE IN A WAVEGUIDE LOADED BY GRADIENT LAYERS}

The spatial structure of the $\mathrm{TE}_{11}$ mode is described by Eqs. (2), (4), and (5); the function $\Psi_{s}(z)$ in these equations is governed by Eqs. (10) and (21). Introducing in Eq. (A3) the definition $2 p=-l(l>0), l=\sqrt{-h^{2}}$, and using the functions in Eq. (A5), one can write the explicit solution of the wave equation (21) for the gradient layer (16) as a sum of forward and backward waves inside the gradient layer:

$$
\Psi_{s}=C_{s}[\cosh (\varsigma)]^{l-1 / 2}\left(F_{1}+Q_{s} F_{2}\right)
$$

where $C_{S}$ is some normalization constant; the parameter $Q_{s}$ determines the contribution of the backward wave to the entire field; and the values of the parameters $\alpha$ and $\beta$ in the functions $F_{1}$ and $F_{2}$ (A5) are given in Eq. (A4): $\alpha=\alpha_{s}, \beta=\beta_{s}$. Substitution of this function $\Psi(z)$ into Eqs. (2), (4), and (5) gives the transverse components of the fields $\vec{E}$ and $\vec{H}$; using these components and taking into account the correlation (6), one can calculate the longitudinal component $H_{z}$ from the first of Maxwell's equations (1):

$$
H_{z}=-\frac{A_{1} \pi\left(a^{2}+b^{2}\right) \Psi_{s}}{a^{2} b} \cos \left(\frac{\pi x}{a}\right) \cos \left(\frac{\pi y}{b}\right) .
$$

Consider the radiation flow of the $\mathrm{TE}_{11}$ mode incident on the boundary $z=0$ of the gradient layer. To find the complex reflection coefficient $R$ for the $\mathrm{TE}_{11}$ mode, we use the continuity conditions for the components $E_{x}, H_{y}$, and $H_{z}$ on the plane $z=0$. Calculations with the components $E_{y}$, $H_{x}$, and $H_{z}$ give the same result. The components $H_{y}$ and $H_{x}$ are expressed via the derivatives of function $\Psi_{s}$ (B1) with respect to the variable $z$ (2), which can be calculated by means of the formulas

$$
\frac{d \varsigma}{d z}=\frac{\cosh (\varsigma)}{L}, \quad \frac{d v}{d z}=-\frac{1}{2 L \cosh (\varsigma)}
$$

Noting that the value of the argument $v$ of the hypergeometric function $G\left(\alpha_{s}, \beta_{s}, \gamma, \nu\right)$ at the plane $z=0$ is equal 
to $v_{+}(\mathrm{A} 6)$, we introduce the notations

$$
\begin{aligned}
& G_{1}=G\left(\alpha_{s}, \beta_{s}, \gamma, \nu_{+}\right), \\
& G_{2}=G\left(\alpha_{s}, \beta_{s}, \gamma, \nu_{-}\right), \quad \nu_{-}=1-v_{+} .
\end{aligned}
$$

Using the expression of the field $\Psi_{s}(z)$ inside the waveguide in the area $z \leq 0$ (8), one can write the continuity conditions for the components $E_{x}, H_{y}$, and $H_{z}$ on the plane $z=0$. These conditions give the equation governing the complex reflection coefficient $R$ :

$$
\begin{gathered}
\frac{1+R_{s 1}}{i k_{z}\left(1-R_{s 1}\right)}=\frac{2 L \sqrt{1+M^{2}}}{-M \sqrt{1+M^{2}}(2 l-1)-\Lambda_{1}} \\
\Lambda_{1}=\frac{G_{1}^{\prime}-Q_{s} G_{2}^{\prime}}{G_{1}+Q_{s} G_{2}} \\
G_{1}^{\prime}=\left.\frac{d G\left(\alpha_{s}, \beta_{s}, \gamma, \nu\right)}{d \nu}\right|_{\nu=\nu_{+}}, \\
G_{2}^{\prime}=\left.\frac{d G\left(\alpha_{s}, \beta_{s}, \gamma, \nu\right)}{d \nu}\right|_{\nu=\nu_{-}} .
\end{gathered}
$$

From Eq. (B5), the expression for $R_{s 1}$ is

$$
\begin{aligned}
R_{s 1} & =\frac{\sqrt{1+M^{2}}\left[2 i L k_{z}+M(2 l-1)\right]+\Lambda_{1}}{I_{s}-\Lambda_{1}}, \\
I_{s} & =\sqrt{1+M^{2}}\left[2 i L k_{z}-M(2 l-1)\right] .
\end{aligned}
$$

The unknown parameter $Q_{s}$ has to be determined from the continuity conditions at the far side of the gradient layer, $z=d$. Taking into account formulas (B5) and (B7), these conditions can be written as

$$
i k_{z}=\frac{M \sqrt{1+M^{2}}(2 l-1)\left(G_{2}+Q_{s} G_{1}\right)+G_{2}^{\prime}-Q_{s} G_{1}^{\prime}}{2 L \sqrt{1+M^{2}}\left(G_{2}+Q_{s} G_{1}\right)} .
$$

Substitution of the value of $Q_{s}$, found from Eq. (B8),

$$
Q_{s}=\frac{G_{2}^{\prime}-I_{s} G_{2}}{G_{1}^{\prime}+I_{s} G_{1}}
$$

into the definition of parameter $\Lambda_{1}$ (B5) gives the value of $\Lambda_{1}$ for the mode $\mathrm{TE}_{11}$ :

$$
\Lambda_{1}=\frac{\left(G_{1}^{\prime}\right)^{2}-\left(G_{2}^{\prime}\right)^{2}-I_{s}\left(G_{1} G_{1}^{\prime}+G_{2} G_{2}^{\prime}\right)}{G_{1} G_{1}^{\prime}+G_{2} G_{2}^{\prime}-I_{s}\left[\left(G_{1}\right)^{2}-\left(G_{2}\right)^{2}\right]} .
$$

Finally, substitution of $\Lambda_{1}$ (B10) into Eq. (B7) results in the explicit formula for the complex reflection coefficient $R_{S 1}$ describing the reflectance of the $\mathrm{TE}_{11}$ mode from the single gradient layer. Substitution of the value of $R_{s 1}(\mathrm{~B} 7)$ into the continuity conditions on the plane $z=d$ gives the complex transmission coefficient $T_{s 1}$ describing the amplitude and phase of the $\mathrm{TE}_{11}$ mode traveling through one gradient layer:

$$
\begin{aligned}
T_{s 1} & =\left|T_{s 1}\right| \exp \left(i \varphi_{s 1}\right) \\
& =\frac{\left(1+R_{s 1}\right)\left[G_{1} G_{1}^{\prime}+G_{2} G_{2}^{\prime}-\Lambda_{1}\left(G_{1}^{2}-G_{2}^{2}\right)\right]}{G_{1} G_{2}^{\prime}+G_{2} G_{1}^{\prime}} .
\end{aligned}
$$

The generalization of these results to the case of a multilayer barrier $(q>1)$ can be carried out by means of the same algorithm. However, a more cumbersome algebra is necessary. An example of such a calculation for a pair of adjoining layers $(q=2)$ is given in Appendix D.

\section{APPENDIX C: PROPAGATION OF THE TH 11 MODE IN A WAVEGUIDE LOADED BY GRADIENT LAYERS}

The reflection of the $\mathrm{TH}_{11}$ mode in a waveguide loaded by gradient layers (16) can be examined by analogy with the analysis developed above in Appendix $\mathrm{B}$ for the $\mathrm{TE}_{11}$ mode. We start, instead of Eq. (B1), from the generating function $\Psi_{p}$,

$$
\Psi_{p}=C_{p}[\cosh (\varsigma)]^{l+1 / 2}\left(V_{1}+Q_{p} V_{2}\right),
$$

where $C_{p}$ is some normalization constant and $V_{1}$ and $V_{2}$ are the solutions of Eq. (21) given by the hypergeometric functions

$$
V_{1}=G\left(\alpha_{p}, \beta_{p}, \gamma, \nu\right), \quad V_{2}=G\left(\alpha_{p}, \beta_{p}, \gamma, 1-v\right),
$$

with the parameters $\alpha_{p}, \beta_{p}$, and $\gamma$ defined in Eqs. (A3) and (A4). We can determine by means of Eq. (2) the transverse components of the $\mathrm{TH}_{11}$ mode $E_{x}, y, H_{x}$, and $H_{y}$. Substituting the components $H_{x}$ and $H_{y}$ into the second of Maxwell's equations (1) and using the condition in Eq. (7) gives the value of the longitudinal component $E_{z}$ :

$$
E_{z}=-\frac{B_{1} \pi\left(a^{2}+b^{2}\right) \Psi_{p}}{a^{2} b} \sin \left(\frac{\pi x}{a}\right) \sin \left(\frac{\pi y}{b}\right) .
$$

Now, the continuity conditions on the plane $z=0$ for the components $E_{x}, H_{y}$, and $E_{z}$ correspond to

$$
\begin{aligned}
\frac{1+R_{p 1}}{i k_{z}\left(1-R_{p 1}\right)} & =-\frac{2 L n_{0}^{2} \sqrt{1+M^{2}}}{M \sqrt{1+M^{2}}(2 l+1)-\Lambda_{1}}, \\
\Lambda_{1} & =\frac{Y_{1}^{\prime}-Q_{p} Y_{2}^{\prime}}{Y_{1}+Q_{p} Y_{2}}
\end{aligned}
$$

The reflection coefficient on the single layer is determined by Eq. (C4). Here, $Y_{1}$ and $Y_{2}$ can be viewed as the result of 
replacing $\alpha_{s}$ by $\alpha_{p}$ and $\beta_{s}$ by $\beta_{p}$ in Eqs. (B4) and (B6) that give the values of $G_{1}, G_{2}$, and their derivatives. We have, now,

$$
\begin{aligned}
& Y_{1}=G\left(\alpha_{p}, \beta_{p}, \gamma, \nu_{+}\right), \quad Y_{2}=G\left(\alpha_{p}, \beta_{p}, \gamma, \nu_{-}\right), \\
& Y_{1}^{\prime}=\left.\frac{d G\left(\alpha_{p}, \beta_{p}, \gamma, v\right)}{d v}\right|_{\nu=v_{+}} \\
& Y_{2}^{\prime}=\left.\frac{d G\left(\alpha_{p}, \beta_{p}, \gamma, v\right)}{d v}\right|_{\nu=\nu_{-}} .
\end{aligned}
$$

Proceeding in a similar way, one can calculate the parameter $Q_{p}$ from the continuity condition at the plane $z=d$ and define the parameter $\Lambda_{1}(\mathrm{C} 4)$ for the $\mathrm{TH}_{11}$ mode:

$$
\begin{aligned}
\Lambda_{1} & =\frac{\left(Y_{1}^{\prime}\right)^{2}-\left(Y_{2}^{\prime}\right)^{2}-I_{p}\left(Y_{1} Y_{1}^{\prime}+Y_{2} Y_{2}^{\prime}\right)}{Y_{1} Y_{1}^{\prime}+Y_{2} Y_{2}^{\prime}-I_{p}\left[\left(Y_{1}\right)^{2}-\left(Y_{2}\right)^{2}\right]} \\
I_{p} & =\sqrt{1+M^{2}}\left[2 i n_{0}^{2} L k_{z}-M(2 l+1)\right] .
\end{aligned}
$$

Finally, the complex reflection coefficient for the $\mathrm{TH}_{11}$ mode passing through a single gradient layer corresponds to

$$
\begin{aligned}
T_{p 1} & =\left|T_{p 1}\right| \exp \left(i \varphi_{p 1}\right) \\
& =\frac{\left(1+R_{p 1}\right)\left[Y_{1} Y_{1}^{\prime}+Y_{2} Y_{2}^{\prime}-\Lambda_{1}\left(Y_{1}^{2}-Y_{2}^{2}\right)\right]}{Y_{1} Y_{2}^{\prime}+Y_{2} Y_{1}^{\prime}} .
\end{aligned}
$$

Note that the expressions for the quantities $\Lambda_{1}, I_{p}$ (C6), and the transmission coefficient for the $\mathrm{TH}_{11}$ mode $(\mathrm{C} 7)$ can be obtained from the corresponding expressions for the $\mathrm{TE}_{11}$ mode exchanging $G_{1}$ by $Y_{1}, G_{2}$ by $Y_{2}$, and replacing $L$ by $L n_{0}^{2}$ and $2 l-1$ by $2 l+1$ in Eq. (B7).

The example of computing the reflection and/or transmission coefficients for the $\mathrm{TH}_{11}$ mode traveling through the pair of gradient barriers is given in Appendix D.

\section{APPENDIX D: TRANSMITTANCE THROUGH A PAIR OF ADJOINING BARRIERS}

To find the complex transmission coefficient for the waves passing through a pair of similar adjoining barriers, one can follow the computational scheme developed above for a single barrier. Assigning the value $q=1$ to the gradient layer at the far side of this structure, we have to examine the reflection of the wave incident on the layer with value $q=2$. The standard continuity conditions on the boundary between layers 1 and 2 have to be taken into account.

Consider, firstly, the propagation of the $\mathrm{TE}_{11}$ mode. Let us introduce the parameter $\Lambda_{2 s}$ analogous to parameter $\Lambda_{1}(\mathrm{~B} 5)$ :

$$
\Lambda_{2 s}=\frac{G_{2}^{\prime}-Q_{2 s} G_{1}^{\prime}}{G_{2}+Q_{2 s} G_{1}} .
$$

Here, the coefficient $Q_{2 s}$ describes, according to Eq. (B1), the contribution of the backward wave to the entire field in layer 2. The continuity conditions on the boundary between the adjoining layers 1 and 2 give the correlation

$$
\Lambda_{2 s}=2 M \sqrt{1+M^{2}}(2 l-1)+\Lambda_{s 1} .
$$

The value $\Lambda_{s 1}$ is defined as $\Lambda_{1}$ in Eq. (B10). Omitting the intermediate calculations, similar to those performed in Appendix B, we find the reflection and/or transmission coefficients for the $\mathrm{TE}_{11}$ mode passing through the pair of barriers:

$$
\begin{gathered}
R_{s 2}=\frac{\sqrt{1+M^{2}}\left[2 i L k_{z}+M(2 l-1)\right]+\chi_{s 2}}{I_{s}-\chi_{s 2}}, \\
\chi_{s 2}=\frac{P_{s} \Lambda_{s 2}+K_{s}}{P_{s}+H_{s} \Lambda_{s 2}}, \quad P_{s}=G_{1} G_{1}^{\prime}+G_{2} G_{2}^{\prime}, \\
K_{s}=\left(G_{1}^{\prime}\right)^{2}-\left(G_{2}^{\prime}\right)^{2}, H_{s}=G_{1}^{2}-G_{2}^{2}, \\
T_{s 2}=\left|T_{s 2}\right| \exp \left(i \varphi_{s 2}\right)=\frac{\left(1+R_{s 2}\right)\left(P_{s}-H_{s} \Lambda_{s 2}\right)}{P_{s}+H_{s} \Lambda_{s 2}} .
\end{gathered}
$$

The corresponding similar expressions obtained for the $\mathrm{TH}_{11}$ mode are

$$
\begin{gathered}
R_{p 2}=\frac{\sqrt{1+M^{2}}\left[2 i n_{0}^{2} L k_{z}+M(2 l+1)\right]+\chi_{p 2}}{I_{p}-\chi_{p 2}}, \\
I_{p}=\sqrt{1+M^{2}}\left[2 i n_{0}^{2} L k_{z}-M(2 l+1)\right], \\
\chi_{p 2}=\frac{P_{p} v+K_{p}}{P_{p}+H_{p} \Lambda_{p 2}}, \quad P_{p}=Y_{1} Y_{1}^{\prime}+Y_{2} Y_{2}^{\prime}, \\
K_{p}=\left(Y_{1}^{\prime}\right)^{2}-\left(Y_{2}^{\prime}\right)^{2}, H_{p}=Y_{1}^{2}-Y_{2}^{2}, \\
\Lambda_{2 p}=2 M \sqrt{1+M^{2}}(2 l+1)+\Lambda_{p 1},
\end{gathered}
$$

with the value $\Lambda_{p 1}$ as defined in Eq. (C6). Finally,

$$
T_{p 2}=\left|T_{p 2}\right| \exp \left(i \varphi_{p 2}\right)=\frac{\left(1+R_{p 2}\right)\left(P_{p}-H_{p} \Lambda_{p 2}\right)}{P_{p}+H_{p} \Lambda_{p 2}} .
$$

[1] A. Ranfagni, P. Fabeni, G. P. Pazzi, and D. Mugnai, Anomalous pulse delay in microwave propagation: A plausible connection to the tunneling time, Phys. Rev. E 48, 1453 (1993). 
[2] V. S. Olkhovsky, E. Recami, and J. Jakiel, Unified time analysis of photon and particle tunneling, Phys. Rep. 398, 133 (2004).

[3] J. D. Baena, L. Jelinek, R. Marqués, and F. Medina, Nearperfect tunneling and amplification of evanescent electromagnetic waves in a waveguide filled by a metamaterial: Theory and experiments, Phys. Rev. B 72, 075116 (2005).

[4] J. B. Pendry, Negative Refraction Makes a Perfect Lens, Phys. Rev. Lett. 85, 3966 (2000).

[5] R. Marqués, J. Martel, F. Mesa, and F. Medina, LeftHanded Media Simulation and Transmission of Electromagnetic Waves in Sub Wavelength Split Ring ResonatorLoaded Metallic Waveguide, Phys. Rev. Lett. 89, 183901 (2002).

[6] M. G. Silveirinha, and N. Engheta, Tunneling of Electromagnetic Energy through the Sub Wavelength Channels and Bends using Epsilon-Near-Zero Metamaterial, Phys. Rev. Lett. 97, 157403 (2006).

[7] R. Liu, Q. Cheng, T. Hand, J. J. Mock, T. J. Cui, S. A. Cummer, and D. R. Smith, Experimental Demonstration of Electromagnetic Tunneling through an Epsilon-Near-Zero Metamaterial at Microwave Frequencies, Phys. Rev. Lett. 100, 023903 (2008).

[8] B. Edwards, A. Alu, M. E. Young, M. Silveirinha, and N. Engheta, Experimental Verification of Epsilon-Near-Zero Metamaterial Coupling and Energy Squeezing, Phys. Rev. Lett. 100, 033903 (2008).

[9] R. Liu, C. Hu, Z. Zhao, and X. Luo, Multipassband effect in multilayered epsilon-near-zero metamaterial, Opt. Express 17, 12183 (2009).

[10] J. B. Pendry, A. J. Holden, W. J. Stewart, and I. Youngs, Extremely Low Frequency Plasmons in the Metallic Mesostructures, Phys. Rev. Lett. 76, 4773 (1996).

[11] O. F. Siddique, O. M. Ramahi, and M. Kashanianfard, Frequency-selective energy tunneling in wire-loaded narrow waveguide channels, Prog. Electromagnet. Res. Lett. 15, 153 (2010).

[12] Y. Li, I. Liberal, C. Della Giovampaola, and N. Engheta, Waveguide metatronics: lumped circuitry, based on structural dispersion, Sci. Adv. 2, e1501790 (2016).
[13] A. B. Shvartsburg, and N. V. Silin, Propagation of microwaves in gradient transmission line: Exactly solvable model, Phys. Scr. 90, 088012 (2015).

[14] D. Pozar, Microwave Engineering (John Wiley \& Sons, Hoboken, 2011), 4th ed.

[15] S. R. Kennedy, and M. J. Brett, Porous broadband antireflection coating by glancing angle deposition, Appl. Opt. 42, 4573 (2003).

[16] A. B. Shvartsburg, Yu. A. Obod, and O. D. Volpian, in Progress in Optics, edited by E. Wolf (2015), Vol. 60, p. 489.

[17] T. K. Kormilina, E. A. Stepanidenko, S. A. Cherevkov, A. Dubavik, M. A. Baranov, A. V. Fedorov, A. V. Baranov, Y. K. Gun'ko, and E. V. Ushakova, A highly luminescent porous metamaterial based on a mixture of gold and alloyed semiconductor nanoparticles, J. Mater. Chem. C 6, 5278 (2018).

[18] A. B. Shvartsburg, and A. A. Maradudin, Waves in Gradient Metamaterials (World Scientific, Singapore, 2013).

[19] L. D. Landau, and E. M. Lifshitz, Quantum Mechanics (Pergamon Press, Bristol, 1984).

[20] I. Bloch, Exploring quantum matter with ultracold atoms in optical lattices, J. Phys. B: At. Opt. Mol. Phys. 38, 629 (2005).

[21] V. C. Aguilera-Navarro, H. Iwamoto, and V. M. de Aquino, Tunneling through rectangular plus linear barrier, Int. J. Theor. Phys. 43, 483 (2004).

[22] M. Li, X. Liu, J. Zhang, X. Ma, X. Sun, Y. Li, and P. Gu, Rigorous analysis of the spatial dispersion effect in multilayer thin-film coatings, J. Opt. Soc. Am A Opt. Image Sci. Vis. 24, 2328 (2007).

[23] J. R. Willis, Exact effective relations for dynamics of a laminated body, Mech. Mater. 41, 385 (2009).

[24] L. Vázquez, S. Jiménez, and A. B. Shvartsburg, The wave equation: From eikonal to anti-eikonal approximation, Mod. Electron. Mater. 2, 51 (2016).

[25] D. Hayes, P. S. Julienne, and I. H. Deutsch, Quantum Logic via the Exchange Blockade in Ultracold Collisions, Phys. Rev. Lett. 98, 070501 (2007).

[26] M. Abramowitz, and I. Stegun, Handbook of Mathematical Functions (Washington D.C., 1968). 